MATEC Web of Conferences 22,03018 (2015)

DOI: $10.1051 /$ matec conf/ 20152203018

(C) Owned by the authors, published by EDP Sciences, 2015

\title{
Study on the Steel Rail Rolling Contact Stress with Consideration of Ini- tial Residual Stress
}

\author{
Hua Song* \& Jian Yang \\ College of Mechanical Engineering and Automation, University of Science and Technology Liaoning, Liaoning, \\ Shandong, China \\ Linbang Song \& Jinkai Yao \\ Shangdong Kerui Petroleum Equipment Co, .Ltd, Dongying, Shandong, China \\ Xinglong Du \\ College of Mechanical Engineering and Automation, University of Science and Technology Liaoning, Liaoning, \\ Shandong, China
}

Wei Li

China Academy of Railway Sciences, Beijing, China

\begin{abstract}
Based on ANSYS/LS-DYNA explicit analysis software, this paper established the three-dimensional finite element model of wheel/rail rolling contact in curve negotiation with consideration of the residual stress impact after rail straightening process. It used the dynain file method to apply the initial residual stress to the rails, and conducted numerical simulation to the process of dynamic wheel/rail rolling contact under the operation condition which is corresponding to the setting of taking velocity and axle load as the variables. The simulation results can show that with the increase of velocity, the equivalent stress on the outer rail railhead and at the rail web will increase accordingly; the equivalent stress on the inner rail railhead and at the rail web will decrease accordingly; and the equivalent stress on the rail flange will be basically the same. With the increase of axle load, all the equivalent stress on the railheads and rail web of the inner rail and the outer rail will increase accordingly while the equivalent stress on the rail flange will be basically the same.
\end{abstract}

Keywords: wheel/rail rolling contact; residual stress; curve negotiation; numerical simulation

\section{INTRODUCTION}

After rolling, cooling, and straightening, the residual stress will stay inside the steel rails, and the usability of the rails will be greatly affected by the residual stress ${ }^{[1]}$. Excessive residual stress remained inside the rails can lead to rail fatigue and fracture or accelerate their development, and thus may cause serious threat to railway running safety. Considering the strength of track structure, the strength of curve track is a weak part. As a result, it is very necessary to study the change laws of the curve rails with initial residual stress under wheel/rail rolling function.

At present, the study of wheel/rail rolling contact problem has been completed widely and deeply. Reference [2] assumed a wheel is of a cylinder shape. On the premise of ignoring the elastic support of rails, the reference studied the normal contact pressure of rails and the distribution of tangential traction under different axle loads and at different speeds. Reference [3] accomplished a full constraint procedure while establishing the finite element model of wheel/rail. It simulated wheel/rail sliding friction in different situations, such as in-situ slipping and complete braking. It also studied the influences that contact status, axle load, slipping velocity, load type and radius of rail tip circle have on the contact stress. Reference [4] applied MARC software to study the wheel/rail contact area, distribution of contact stress, and deformation in the situation of no elastic support from sleeper. Reference [5] set the track support as a rigid body. It did full constraint on both ends and the bottom of the steel rails; established the finite element model of three-dimensional steel rail elasticity rolling contact; and analyzed the influence that rail material yield strength has on the residual stress and strain of the rails. References [6-8] established the finite element model of wheel/rail contact, and did research on wheel/rail contact problems. However, in order to simplify the analysis, the track model used in the references was a straight track. Reference [9] did research on the wheel/rail contact of curve track. It applied constant transverse force to replace the centrifugal force generated by wheels in practical situation.

To sum up, nowadays, there have been plenty research on the wheel/rail contact problems of straight track. But the research on curve tracks which are weak in strength and easy to be damaged is very little. The

*Corresponding author: songhua88@126.com

This is an Open Access article distributed under the terms of the Creative Commons Attribution License 4.0, which permits unrestricted use, distribution, and reproduction in any medium, provided the original work is properly cited. 


\section{MATEC Web of Conferences}

existing research on curve tracks mainly focuses on the contact of single track with single wheel. No consideration has been taken into the influence left by the residual stress generated during the steel rail manufacturing. Taking the status of the residual stress generated during the steel rail manufacturing into consideration, this paper applied ANSYS/LS-DYNA to establish the three-dimensional wheel/rail rolling contact model of curve track. It completed a dynamic simulation and did research on the impact that velocity and axle load have on the wheel/rail contact position and the steel rail stress status which can provide scientific proof for the stress status required in steel rail fatigue wear studies, and can also offer related reference for track design.

\section{FINITE ELEMENT MODEL OF WHEEL/RAIL ROLLING CONTACT}

\subsection{Equivalent stiffness computation}

The equivalent stiffness of rail ${ }^{[10]}$ is composed of three parts: fastener stiffness, spacer stiffness and ballast bed stiffness. Fasteners, spacers and ballast bed are laid from up to down in series. The equivalent stiffness of rail $k_{\mathrm{z}}$ is shown as follows

$$
k_{z}=1 /\left(\frac{1}{k_{1}}+\frac{1}{k_{2}}+\frac{1}{k_{3}}\right)
$$

In this equation, $k_{1}$ refers to the fastener stiffness, $k_{2}$ refers to the spacer stiffness, and $k_{3}$ refers to the ballast bed stiffness.

\subsubsection{Fastener stiffness computation}

The most commonly-used fastener types in domestic railways are $X$ spring bar fastener, gasket fastener, type III spring bar fastener and heightening type fastener. Take the gasket fastener as an example, it is made up by gasket, coil spring and bolt. As the elasticity of fastener is provided by coil spring, the coil spring stiffness is the stiffness of gasket fastener. The computing equation of fastener stiffness $k_{1}$ is given as follows:

$$
k_{1}=\frac{G d^{4}}{64 R^{3} n}
$$

In this equation, $G$ refers to the shear modulus of steel, $n$ refers to the number of effective spring coils, $R$ refers to the radius of spring (from the center of spring to the center of spring bar), and $d$ refers to the diameter of spring bar.

\subsubsection{Spacer stiffness computation}

The commonly-used gaskets under rails are rubber gaskets which are mainly in rectangle shape. The loading area of a rubber gasket is $A_{\mathrm{L}}=a b$ ( $a$ and $b$ respectively refer to the length and width of the rectan- gle) while $H$ refers to the compressed height of rubber gasket. The stiffness of rectangular rubber gasket $k_{2}$ can be expressed as follows:

$$
k_{2}=\frac{A_{L} G}{H\left[1+0.29(H / b)^{2}\right]}
$$

\subsubsection{Ballast bed stiffness computation}

The ballast bed stiffness is related to the elasticity modulus of ballast ${ }^{[11]}$. As the force in ballast bed expends downward by the internal friction angle $\psi$ of ballast, the ballast stress in unit area will be decreased accordingly. Set $l$ and $n$ as the length and width of the contact face for the sleeper and the ballast bed, the actual loaded face of ballast bed is $s=(l+c z)(n+c z)$. From Reference [12], it can be seen that the ballast bed stiffness $k_{3}$ can be expressed as follows:

$$
k_{3}=\frac{E_{3} S}{z}
$$

After conducting differential procedures in Equation (4), it can be obtained that $d k_{3} d z=E_{3} d x d y$.

The results of integration on both sides are shown as follows:

$\iint d k_{3} d z=E_{3}(l+c z)(n+c z)$

$E_{3}=k_{3} \frac{1}{c(l-n)} \ln \left[\frac{l(n+c z)}{n(l+c z)}\right]$

And then, the ballast bed stiffness $k_{3}$ can be expressed as follows:

$$
k_{3}=\frac{c(l-n) E_{3}}{\ln \left[\frac{l}{n} \frac{(n+c z)}{(l+c z)}\right]}
$$

In this equation, $c$ refers to $2 \operatorname{tg} \psi, z$ refers to the coordinate of ballast bed depth, and $E_{3}$ refers to the elasticity modulus of ballast.

As shown in Table 3 (List of the Vertical Models of Common Rail Carriages) in the Appendix 3 of Reference [13], the values of fastener stiffness $k_{1}$, the spacer stiffness $k_{2}$ and the ballast bed stiffness $k_{3}$ can be found in Table 1 given below. Thus, the equivalent stiffness of the track is $k_{\mathrm{z}}=1 /\left(1 / k_{1}+1 / k_{2}+1 / k_{3}\right)=3612.9032 \mathrm{~N} / \mathrm{mm}$

Table 1. Track parameter values

\begin{tabular}{lll}
\hline Parameter & Value & Unit \\
\hline Fastener stiffness $k_{1}$ & $3.85 \mathrm{e} 3$ & $\mathrm{~N} / \mathrm{mm}$ \\
Spacer stiffness $k_{2}$ & $8 \mathrm{e} 4$ & $\mathrm{~N} / \mathrm{mm}$ \\
Ballast bed stiffness k3 & $2.2 \mathrm{e} 5$ & $\mathrm{~N} / \mathrm{mm}$
\end{tabular}




\subsection{Modeling}

The type of used wheels is a worn-type (LM) with diameter of $840 \mathrm{~mm}$. The rail type is $75 \mathrm{~kg} / \mathrm{m}$ with length of $5 \mathrm{~m}$. The texture of rail material is $\mathrm{U} 75 \mathrm{~V}$ and the track gauge is $1450 \mathrm{~mm}$. The wheels shall be set as the rigid body. The related material parameters of the rails are shown in Table 2: the yield limit is $525 \mathrm{MPa}$; the shear modulus is $7.9 \mathrm{e} 4 \mathrm{MPa}$; the elasticity modulus is $2.1 \mathrm{e} 5 \mathrm{MPa}$; and the Poisson's ratio is 0.3 . We use the bilinear kinematic hardening to describe the elastic characteristics of the steel rails. The unit type shall be 8 -node Solid164. The face-face penalty function contact algorithm shall be used to describe the rolling contact between wheel and track. To avoid the direct connection of the track and the equivalent springs under the track, the base plate models shall be built between the rail foot surface and each group of vertical equivalent spring under the track, and all nodes on the base plate top surface and the rail foot surface shall be integrated together, so as to simulate the support provided by the rail for the track. We use stiffening bars to connect with the base plates on the inside and outside rail foot surfaces, so as to simulate the connecting function applied by the rail sleepers to the inside and outside rails. In ANSYS/LS-DYNA, as models that made of the same material are not allowed to set up the stiffness and damping of spring unit simultaneously, the stiffness and damping of equivalent spring need to be set up respectively. Therefore, during the process of modeling, two groups of spring unit shall be established in the same position, and both shall be respectively equipped with the stiffness model and the damping material model. The total node quantity of the finite element model of wheel/rail rolling contact is 106228 , and the total unit number is 759200 as shown in Figure 1.

Table 2. Rail parameter values

\begin{tabular}{lll}
\hline Material parameter & Value & Unit \\
\hline Elasticity modulus & $2.1 \mathrm{e} 5$ & $\mathrm{MPa}$ \\
Poisson's ratio & 0.3 & - \\
Yield limit & 525 & $\mathrm{MPa}$ \\
Shear modulus & $7.9 \mathrm{e} 4$ & $\mathrm{MPa}$ \\
\hline
\end{tabular}

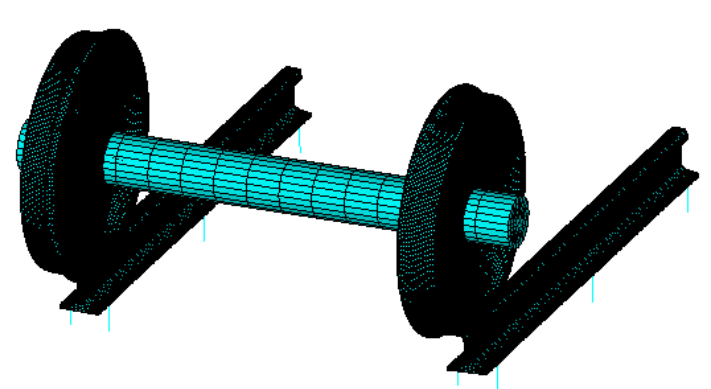

Figure 1. FEM of wheel/rail rolling contact
Apply symmetry constraint to both sides of the rails. Add full constraint of displacement on the rail foot surface. Apply initial rotational velocity to wheel sets along the axial direction, and bring constant rotational velocity to wheel sets while taking the curvature central axis of the curve track as the criterion, so as to simulate the function of centrifugal force. In the meantime, simulate the axle load by applying vertical stress on wheel sets.

\subsection{Import of initial residual stress}

Shanhu Tong ${ }^{[12]}$ established the finite element model of rail compound straightening based on ANSYS/LS-DYNA, and obtained the curve chart of the vertical residual stress on the rail section after straightening as shown in Figure 2.

The residual stress data accords with the regulation set in Railway Standard TB/T2344-2003 and The Technical Specifications of $43 \mathrm{~kg} / \mathrm{m}-75 \mathrm{~kg} / \mathrm{m}$ Hot Rolled Rail Ordering, namely the maximum vertical residual stress on rail flange shall be less than $250 \mathrm{MPa}$.

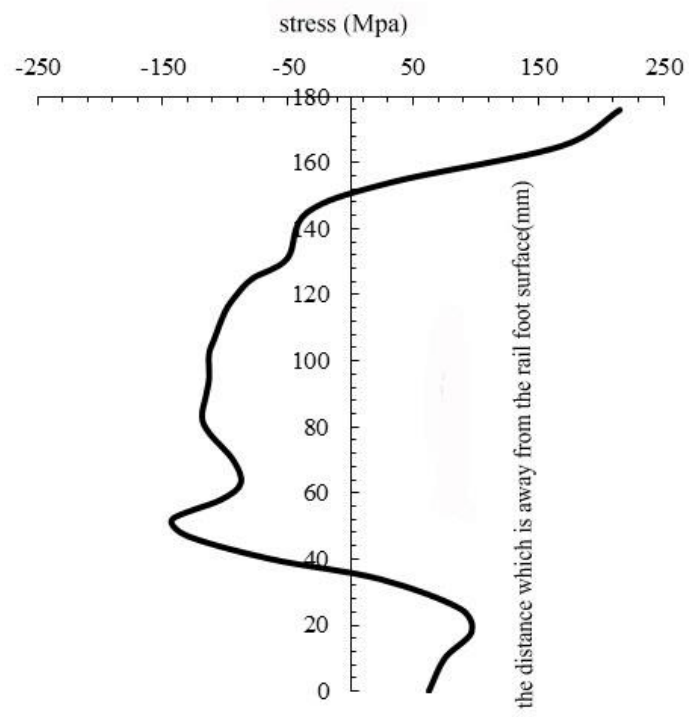

Figure 2. Initial residual stress of rail section

Use the dynain document method to export the data of rail residual stress after straightening from the prosprocessor LS-PrePost of LS-DYNA by format of dynain ascii. Extract the parts containing the key words *INITIAL_STRESS_SOLID of LS-DYNA and add them to the $\mathrm{K}$ file generated by the finite element model of wheel/rail rolling contact. The initial residual stress of rail after straightening can be imported in this way as shown in Figure 3. 


\section{MATEC Web of Conferences}
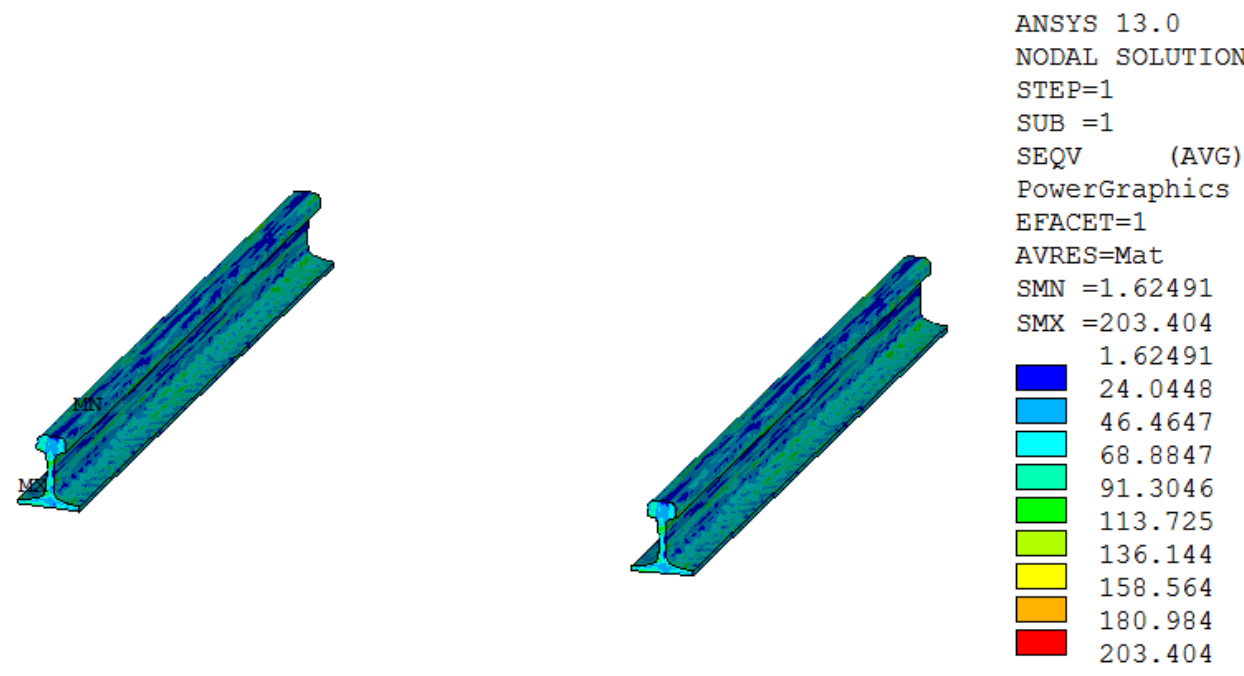

Figure 3. Cloud chart of rail initial residual stress

\section{ANALYSIS OF NUMERICAL SIMULATION RESULT}

\subsection{Determination of simulation scheme}

In order to present the internal stress state of rails based on the function of wheel rolling and the influences that the track curvature radius, the excessive height, the rail cant, the wheel velocity and the axle load have on the rails, corresponding studies have been done to analyze the stress state of the rails at different speeds $(100 \mathrm{~km} / \mathrm{h}, 150 \mathrm{~km} / \mathrm{h}$ and $200 \mathrm{~km} / \mathrm{h})$ and with different axle loads $(21 t, 28 t$ and $35 t)$ : Set track radius of $1000 \mathrm{~m}$, excessive height of $80 \mathrm{~mm}$, axle load of $28 \mathrm{t}$ and velocity of $150 \mathrm{~km} / \mathrm{h}$ as the basic simulation parameters.

\subsection{Influence on rail stress state left by velocity and} axle load

The Von Mises stresses mentioned in this paper were all taken from the 25 nodes on the symmetric line of rail section in order to analyze the influence on rail stress state left by velocity and axle load.

3.2.1 Influence on rail stress state left by velocity Take the Von Mises stresses of the nodes on the symmetric line of rail section as the foundation. Respectively draw up the change curves of Von Mises residual stress at different speeds $(100 \mathrm{~km} / \mathrm{h}, 150 \mathrm{~km} / \mathrm{h}$ and $200 \mathrm{~km} / \mathrm{h}$ ) as shown in Figure 4 and Figure 5.

From Figure 4 and Figure 5, it can be seen that with the increase of velocity and the equivalent stress values of the nodes on outer rail heads and rail web section, the equivalent stress values on inner rail heads and rail web section will be decreased while the equivalent stress values of the nodes on rail foot section will basically remain the same. The reason for

this phenomenon is that with the increase of velocity and vehicle centrifugal force, wheel sets will shift outwards and thus make outer rail stress bigger and inner rail stress smaller.

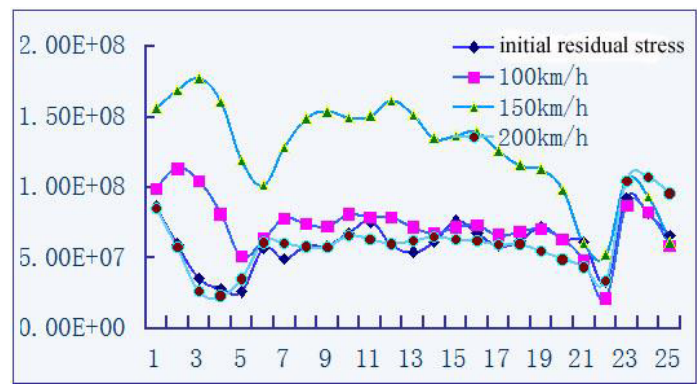

Figure 4. Von Mises stress of outer rail at different speeds

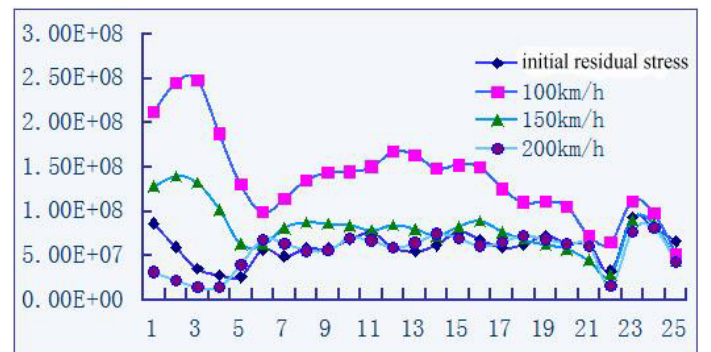

Figure 5. Von Mises stress of inner rail at different speeds

3.2.2 Influence on rail stress state left by axle load Take the Von Mises stresses of the nodes on the symmetric line of rail section as the foundation. Re- 
spectively draw up the change curves of Von Mises residual stress applied to inner rail wheel sets and outer rail wheel sets at different axle loads $(21 \mathrm{t}, 28 \mathrm{t}$ and 35t) as shown in Figure 6 and Figure 7:

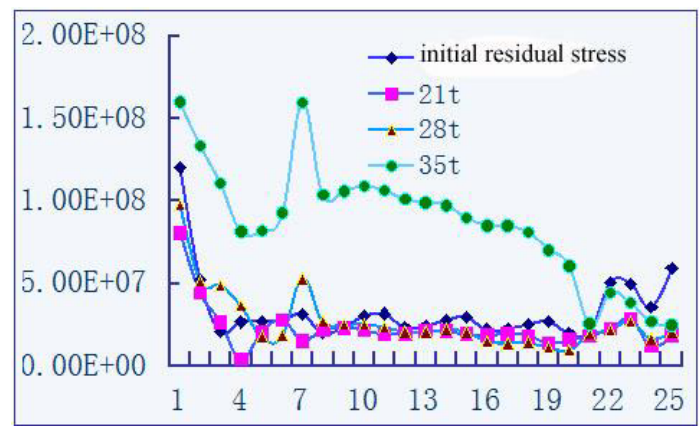

Figure 6. Von Mises stress of outer rail at different axle loads

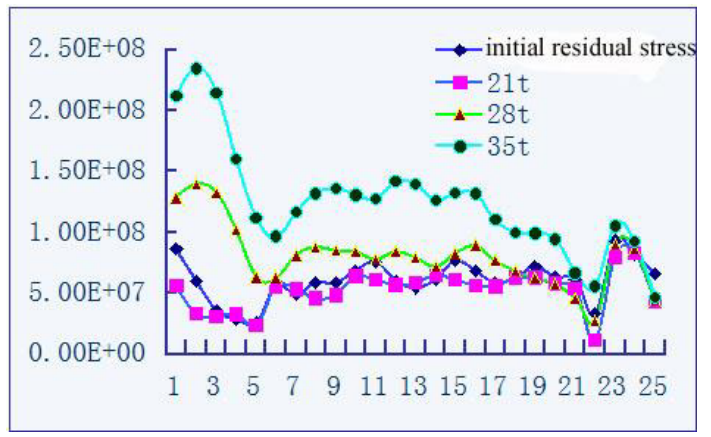

Figure 7. Von Mises stress of inner rail at different axle loads

From Figure 6 and Figure 7, it can be seen that with the increase of axle load, all the equivalent stress on the railheads and rail web of the inner rail and the outer rail will be increased accordingly while the equivalent stress on the rail flange will be basically the same.

\section{CONCLUSION}

With consideration of the residual stress applied after rail straightening process and the influence left by the vertical stiffness of the tracks, the three-dimensional finite element model for the wheel/rail rolling contact on curve tracks has been established based on $A N$ $S Y S / L S-D Y N A$. Through the numerical simulation of the steel rail rolling contact process after applying the initial residual stress, conclusions can be drawn as follows: With the increase of velocity, the equivalent stress on the outer rail railhead and at the rail web will be increased accordingly; the equivalent stress on the inner rail railhead and at the rail web will be decreased accordingly; and the equivalent stress on the rail flange will be basically the same. With the increase of axle load, all the equivalent stress on the railheads and rail web of the inner rail and the outer rail will be increased accordingly while the equivalent stress on the rail flange will be basically the same.

\section{ACKNOWLEDGEMENT}

This project was supported by the National Natural Sc ience Foundation of China (51074088, 51374127).

\section{REFERENCES}

[1] Mi Gumao. 1983. Generation and Countermeasures of Residual Stress. Beijing: Machinery Industry Press.

[2] Jiang Xiaoyu \& Jin Xuesong. 2006. Numerical simulation of wheel rolling over rail at high-speeds. Wear, 262(5): 666-671.

[3] Wang Juan. 2013. Analysis of wheel-rail friction sliding contact stress based on ANSYS. Journal of Machine Design and Research. 124(4):699-708.

[4] Zefeng Wen. 2011. Three-dimensional elastic-plastic stress analysis of wheel-rail rolling contact. Wear, 271(1): 426-436.

[5] Telliskivit, olofsson U. 2001. Contact mechanics analysis of measured wheel/rail profiles using the finite element Method. Journal of Rail and Rapid Transit, 21(5): 65-72.

[6] Zhu Chunhai. 2008. Finite Element Analysis of the Conformal Contact Problem of Wheel/Rail. Journal of Dalian Jiaotong University, 29(3): 9-13.

[7] Otto Kleiner. 2014. Investigating stress on wheels and rails. Foreign Rolling Stock, 10(3): 25-30.

[8] Liu Yingxi. 2005. Analysis of wheel/rail two-point contact by using finite element method. Chinese Journal of Mechanical Engineering. 41(11): 121-126.

[9] Lu Yizhi. 2011. Finite Element Analysis of Contact between the Wheel and the Curve Rail. Journal of East China Jiaotong University. 28(5): 62-66.

[10]Lian Songliang. 2004. Theoretical Calculation of the Track Structure Stiffness. China Railway Science. 25(1):68-71.

[11]Kerr.A .D. 1983. Orbital mechanics and projects. Beijing: China Railway Publishing House.

[12]Lei Xiaoyan, 1998. The Railway Track Structure Numerical Analysis Method. Beijing: China Railway Publishing House.

[13]ZHAI Wanming. 2007. Vehicle-Track Coupling Dynamics (Third Edition). Beijing: Science Press.

[14]Tong Shanhu. 2012. The Numerical Simulation for Hot-Preflex Heavy Rail Straightening. Liaoning: University of Science and Technology Liaoning. 